\title{
Ein Wort-Ton-Kunstwerk im Sinne Richard Wagners? $\mathrm{Zu}$ Hans Pfitzners Lethe
}

Das Lied bildet den Ausgangspunkt in Pfitzners Schaffen und ist zugleich die Gattung, die in seinem CEuvre einen überaus prominenten Platz einnimmt. Während die Lieder wiederholt Gegenstand von Untersuchungen waren, ${ }^{1}$ standen die Orchestergesänge im Allgemeinen - im Gegensatz zu den entsprechenden Werken Mahlers, Strauss', Schönbergs sowie Schoecks - und Lethe im Besonderen bisher kaum im Blickpunkt des wissenschaftlichen Interesses. ${ }^{2}$ Pfitzner bedachte das Orchesterlied, von Bearbeitungen abgesehen, ${ }^{3}$ mit fünf Werken: Herr Oluf op. 12 (1891), das mit zum Beginn der Gattungsgeschichte gehört (1897 entstanden Strauss' Vier Gesänge und 1899 Zwei gräßsere Gesänge), Heinzelmännchen op. 14 (1902/03), Zwei deutsche Gesänge op. 25 (1916) sowie schließlich Lethe op. 37 (1926), womit er nach zehnjähriger Unterbrechung nochmals zum Orchesterlied zurückkehrte und, obwohl die Blütezeit der Gattung eher als vergangen angesehen werden muss, ein, wie ich zu zeigen versuchen möchte, im Vergleich zu seinen früheren Beiträgen gattungsgeschichtlich herausragendes Werk vorlegte, das den Vier Letzten Liedern (1948) von Richard Strauss zumindest im Hinblick auf den ästhetischen Anspruch durchaus nicht nachsteht.

Als eine eigenständige, für das Fin de siècle besonders typische Gattung, deren ästhetischsoziologische Voraussetzungen, wie Danuser ausführlich dargelegt hat, sich deutlich von denen des orchestrierten Liedes unterscheiden, tritt das Orchesterlied erst Ende des 19. Jahrhunderts in Erscheinung. ${ }^{4}$ Den von Danuser benutzten Terminus „Orchestergesang “ hat, soweit ich es sehe, Siegmund von Hausegger in die Diskussion eingebracht, ${ }^{5}$ möglicherweise in Anlehnung an Zuccalmaglio, der mit dem Sprechen vom „Gesang“ statt vom „Lied“ eine neue ästhetische Haltung verbunden wissen wollte: „Im Gesange“, so Zuccalmaglio, „ver-

1 Werner Diez, Hans Pfitzners Lieder. Versuch einer Stilbetrachtung, Regensburg 1968; Wolfgang Osthoff (Hrsg.), Hans Pfitzner und die musikalische Lyrik seiner Zeit. Bericht über das Symposion Hamburg 1989 (= Veröffentlichungen der Hans Pfitzner-Gesellschaft, hrsg. von Wolfgang Osthoff, 6), Tutzing 1994.

2 Während Hans Rectanus' Text Die Orchesterlieder Hans Pfitzners im Kontext seines Liedschaffens (in: Pfitzner, Hindemith, Frankfurt und die Moderne [= Mitteilungen der Hans Pfitzner-Gesellschaft, Neue Folge 71] hrsg. von Rolf Tybout, Tutzing 2011, S. 44-59) als eine überblicksartige Einführung in den entsprechenden Werkkomplex verstanden werden kann, thematisiert Rolf Urs Ringger (Hans Pfitzners Orchesterlied „Lethe“, in: ders., Von Debussy bis Henze. Zur Musik unseres Jahrhunderts, München 1986, S. 94-100) vor allem die Bedeutung des Orchesters und die avancierte Harmonik in Lethe.

3 Pfitzner orchestrierte Schumann- und Loewe-Lieder sowie eigene Lieder (op. 5.1, op. 4,1). Siehe Edward F. Kravitt, Das Lied - Spiegel der Spätromantik [The Lied. Mirror of late Romanticism, Yale 1966], Hildesheim 2004, S. 347, Anm. 27, sowie Rectanus, Die Orchesterlieder Hans Pfitzners, S. 45ff.

4 Hermann Danuser, Der Orchestergesang des Fin de siècle: eine historische und ästhetische Skizze, in: Mf 30 (1977), S. 425-452.

5 Siegmund von Hausegger, Über den Orchestergesang (1912), in: Betrachtungen zur Kunst. Gesammelte Aufsätze (= Die Musik, 39.-41. Bd.), Leipzig o. J. [1921], S. 205-214. 
zichten wir auf die anspruchslose Naivetät“ ${ }^{6}$ Auch Adolf Bernhard Marx vermeidet, indem er das entsprechende Kapitel seiner Kompositionslehre „Gesang mit Orchesterbegleitung “7 überschreibt, den Begriff „Lied“, verweist also auf einen von dieser Gattung unterschiedenen Kontext und antizipiert damit die spätere These Walther Dürrs, dass „Orchesterlieder [...] über den Rahmen der Gattung hinausführen". 8

Der im Wesentlichen von Siegmund von Hausegger und Rudolf Louis geführte gattungsästhetische Diskurs muss hier nicht im Einzelnen wiederholt werden; es genügt der Hinweis, dass von Hausegger im Gegensatz zu Louis das Orchesterlied als eine zukunftsträchtige Gattung sah. Einig waren sich beide in der Ablehnung des im engeren Sinne lyrischen Orchesterlieds, entsteht doch, wie Louis formuliert, ,allzu leicht [...] ein störendes Missverhältnis zwischen der Intimität des Inhalts und der Stärke der in Anspruch genommenen klanglichen Mittel [...]“.9 Kaum zu überhören ist in diesen Worten des promovierten Philosophen Louis die Auffassung G. W. F. Hegels, zu einer wirklichen Verschmelzung von Wort und Musik könne es nur bei Liedern kommen, ,in welchen die Stimmung, das Gemüt das Vorwaltende bleibt, und die Musik nun diesen inneren Klang der Seele zur Melodie zu verstärken und auszubilden hat". ${ }^{10}$

Wie lange ein solches Denken noch wirksam sein konnte, erhellt aus einer Kritik in der London Times nach der Uraufführung der Vier Letzten Lieder von Strauss am 22. Mai 1950 in der Royal Albert Hall:

„as is also case in Mahler's orchestral songs, there seems some disproportion between the magnitude of the medium and the essential intimacy of lyrical utterance". ${ }^{11}$

Da die mit der Gattung zwanghaft verbundene Intimität bei der Ballade ebenso wenig erforderlich ist wie die Identifizierung mit dem Sänger als Träger des lyrischen Ichs, ließ Rudolf Louis konsequenterweise der erzählenden Grundhaltung, des epischen Charakters wegen einzig den balladesken Dichtungstypus als Grundlage von Orchesterliedern gelten ${ }^{12}$ und hob deshalb im Hinblick auf die Orchestergesänge Pfitzners hervor, „dass es ausschließlich balladenartige Dichtungen sind, zu deren Vertonung er den anspruchsvollen Apparat des vollen Orchesters aufbot" ${ }^{13}$ Inwieweit das auch für Lethe Gültigkeit hat, wird noch zu diskutieren sein.

Wenn dem Liedbegriff im 19. Jahrhundert die Aura des Einfachen auch abhandenzukommen scheint - dies zeigt nicht zuletzt die Ausprägung der Polarität von Volks- und Kunstlied -, so bleiben doch die auf Haus und Salon verweisende Innerlichkeit und Intimität Gattungskonstituenten, die beim Vortrag im bürgerlich-öffentlichen Konzertbetrieb durchaus als verletzt angesehen wurden. Damit tut sich ein zweites Problemfeld auf, das aber schon in der zweiten Hälfte des 19. Jahrhunderts Virulenz erlangte, als das Lied mit

6 August Wilhelm von Zuccalmaglio, Das deutsche Lied, in: NZfM 20 (1844), S. 70.

7 Adolf Bernhard Marx, Die Lehre von der Komposition, Bd. IV, Leipzig 51879 , S. 485

8 Walther Dürr, Das deutsche Sololied im 19. Jahrhundert. Untersuchungen zu Sprache und Musik, Wilhemshaven 1984, S. 300.

9 Rudolf Louis, Die deutsche Musik der Gegenwart, München 1909, S. 237.

10 Georg Wilhelm Friedrich Hegel, Vorlesungen über die Ästhetik III, hrsg. von Eva Moldenhauer und Karl Markus Michel, Frankfurt a. M. 1970 [= Werke, Bd. 15], S. 450.

11 Zitiert nach Hans-Joachim Bracht, Nietzsches Theorie der Lyrik und das Orchesterlied, Kassel usw. 1993, S. 77.

12 Louis, Die deutsche Musik, S. $236 f$.

13 Bracht, Nietzsches Theorie, S. 20. 
der Institutionalisierung von Liederabenden ${ }^{14}$ aus der Sphäre des Privaten, des intimen Salons hinaustrat in den Konzertsaal und damit öffentlich wurde. Diesem Strukturwandel der Aufführungssituation trat Brendel, der Apologet der Neudeutschen Schule, mit der These, das Lied als das „nur Subjective“ sei ungeeignet für den öffentlichen Vortrag, ${ }^{15}$ ebenso entgegen wie später noch Hermann Kretzschmar ${ }^{16}$ und Paul Bekker. ${ }^{17}$ Alle Bedenken konnten jedoch nicht verhindern, dass der öffentliche Liederabend gleichberechtigt neben andere geschlossene Programmtypen trat, das Lied also nicht mehr nur als Einlage eines Konzertprogramms diente.

Der ausführlicheren Auseinandersetzung mit Lethe sei ein für die angemessene Bewertung von Pfitzners letztem Beitrag zur Gattung unverzichtbarer kurzer Blick auf die übrigen Orchestergesänge vorangestellt. Wenn auch nicht von einer Verschmelzung von Lied und Symphonie wie im Falle von Gustav Mahlers Lied von der Erde die Rede sein kann, zeigt Pfitzners Gattungserstling Herr Oluf ${ }^{18}$ - die Vertonung einer von Johann Gottfried Herder bearbeiteten und übersetzten dänischen Volksballade, ${ }^{19} 1777 \mathrm{im}$ zweiten Teil der Volkslieder unter dem Titel „Erlkönigs Tochter“ publiziert - im Hinblick auf Formverlauf und Motivdiskurs durchaus symphonisch zu nennende Charakteristika. Die den Strophen 1 bis 12, 13 bis 17 und 18 bis 21 zugeordneten drei Szenerien der Ballade finden sich wieder in den drei durch größere Orchesterzwischenspiele getrennten Abschnitten der Komposition. Während die beiden antagonistischen Themen („Herr Oluf“-Thema und „Erlkönigs Tochter“-Thema) im ersten Teil exponiert werden, kommt dem ersten Orchesterzwischenspiel (als Buchstabe M) mit der Konfrontation der beiden Themen durchaus Durchführungscharakter zu. Die zentralen Themen bzw. motivischen Gestalten beruhen zum großen Teil auf dem Prinzip der kontrastierenden Ableitung, mit dem, ähnlich wie in Franz Liszts Symphonischen Dichtungen, das Auseinanderfallen in „ein Potpourri von Momentaneffekten “20 verhindert werden soll. So greift das „Erlkönigs-Tochter“-Thema (T. 5, Pos.) den gebrochen-aufsteigenden Quartsextakkord des „Herr-Oluf-“Thema (ab dem 3. T. nach Buchstabe C) auf, das seinerseits in zwei Gestalten existiert: Einmal als unverändertes Instrumentalthema und einmal als unverkennbar deutlich aus dem Instrumentalthema abgeleitetes Vokalthema mit verschiedenen Derivaten (z. B. 4 T. vor Buchstabe B). D. h. es gibt keine Motive oder Figuren, die ausschließlich im Orchester oder in der Gesangsstimme zu finden sind. Damit

14 Ulrich Mahlert, Fortschritt und Kunstlied. Späte Lieder Robert Schumanns im Licht der liedästhetischen Diskussion ab 1848 (= Freiburger Schriften zur Musikwissenschaft 13, hrsg. von Hans Heinrich Eggebrecht), München 1983, S. 39.

15 Franz Brendel, Thesen über Concertreform, in: NZfM 45 (1856), S. 97ff., S. 109ff., S. 117ff., S. $129 \mathrm{ff}$.

16 Hermann Kretschmar, Das deutsche Lied seit dem Tode Richard Wagners, in: ders., Gesammelte Aufsätze aus den Jahrbüchern der Musikbibliothek Peters, Reprint der Ausgabe 1911, hrsg. von Karl Heller, Leipzig 1973, S. 27.

17 Paul Bekker, Das Lied. Ein kritisches Fragment (1921), in: ders., Klang und Eros. Zweiter Band der gesammelten Schriften, Stuttgart und Berlin 1922, S. 284-293.

18 Berlin 1902 (Bote \& Bock)

19 Goethe übernahm für seinen Erlkönig daraus das Hauptmotiv, die unheilvolle Begegnung mit dem Jenseitigen, und die Nebenmotive: den nächtlichen Ritt und die steigende Lockung bis zur todbringenden Berührung.

20 Carl Dahlhaus, Liszts Bergsymphonie und die Idee der Symphonischen Dichtung, in: Jahrbuch des Staatlichen Instituts für Musikforschung Preußischer Kulturbesitz, Kassel 1975, S. 96-131, hier: S. 99 
deutet sich in Pfitzner Komposition eine Vereinheitlichung des Satzes in der Vertikalen an, wie sie typisch sein wird z. B. für Mahlers Kindertotenlieder (1901-1904) und für Schönbergs Gurre-Lieder.

Die Heinzelmännchen ${ }^{21}$ ist eine wahrscheinlich auf einer Kölner Sage beruhende volkstümliche, aus der Feder August Kopischs stammende Ballade. Die zum für das Biedermeier typischen naturmagischen Typus ${ }^{22}$ zählende Ballade - sie erzählt die Geschichte von der Schar Heinzelmännchen, die faulen Handwerkern nachts die Arbeit verrichten - besteht aus 8 Strophen mit jeweils 14 Paarreime bildenden Versen, wobei das Versmaß in regelmäßigen Abständen wechselt. Die Zeilen 1-4 und 13/14 jeder Strophe stehen im vierhebigen Jambus, die Zeilen 5-8 sowie 11/12 im zweihebigen Daktylus, und die Zeilen 9/10 ergeben einen einhebigen Daktylus. Die Singstimme ist der solchermaßen streng gebauten Dichtung im Hinblick auf Phrasenbildung und Deklamationsstruktur eng angelehnt. Betrachtet man die die einzelnen Handlungen der Heinzelmännchen aufzählenden Zeilen 7 bis 12 einer jeden Strophe, so zeigt die Vertonung mit dem Aufstellen eines Modells, das aus der Konjunktion „und“ sowie einem Verb bestehende Einheiten durch Pausen trennt, die Tendenz zu einer der Textvorlage korrespondierenden Vereinheitlichung des Parameters Rhythmus. Abweichungen gründen in der jeweiligen syntaktischen Situation: In der 1. Strophe z. B. entfällt die Pause, weil der Anfang der 7. Zeile das Subjekt der 5. Zeile nachliefert, in der 3. Strophe, weil das Verb mit einem Adverb verbunden ist. Löst sich die Singstimme von der Textstruktur, gründet dies vorwiegend in der Intention, semantische Details zu akzentuieren. Hierfür zwei Beispiele. Markant kontrastierend ist die zwölfte Zeile der 7. Strophe gearbeitet: Vorbereitet durch auf eine Gewichtung der Silben verzichtende elfte Zeile, wird das „vermaledeien“ gleich einer exclamatio auf einem Ton mit einer übermäßigen Dehnung der Akzentsilbe regelrecht hinausgeschrien. In den Zeilen neun bis zwölf der 8. Strophe schließlich fallen Versakzent und rhythmischer Akzent auseinander. Das hier aufgestellte neue Deklamationsmuster ist gleichsam die musikalische Umsetzung der in der Partitur notierten Anweisung „missmutig“.

Dass Pfitzner die Textvorlage für eine Reihe von Orchestereffekten nutzt, ist schon häufiger festgestellt worden. Dabei erschöpft sich die Musik keineswegs in „malender“ Darstellung. Wie schon in Herr Oluf verhindert Pfitzner durch einen dichten motivischen Konnex, dass die Komposition in eine bloße Reihung von Tableaux zerfällt. Der erste Takt der Singstimme erweist sich dabei als zentral für die motivische Erfindung. Motivderivate bilden am Ende der 1. Strophe den Übergang zur 2. Strophe sowie den Übergang zur dritten Strophe. Zwischen 4. und 6. Strophe bilden sie ein Ritornell. Am Ende der 1. Strophe ist ein Motivderivat gleichzeitig Antizipation der Begleitung der ersten vier Zeilen der 2. Strophe. Zu Beginn der 4. Strophe bildet es den Kontrapunkt zu den Sechzehntelfigurationen. Und in der 7. Strophe, verkürzt auf getupfte Achtel, erklingt es zwischen dritter und vierter Zeile der 1. Strophe.

Die politisch motivierten Zwei deutsche Gesänge ${ }^{23}$ op. 25, dem „Großadmiral von Tirpitz zugeeignet", auf Gedichte von August Kopisch und Joseph von Eichendorff, stellen sowohl inhaltlich als auch ästhetisch den denkbar größten Gegensatz zu Lethe dar, ist dieses Werk doch, wie noch zu zeigen sein wird, ein zutiefst emotionaler, aufgrund des vielschichtigen Verweischarakters der musikalischen Strukturen hochkomplexer Reflex auf eine

21 Rolandswerth, 2. Aufl. o. J. (Mat. Brockhaus)

22 Vgl. Wolfgang Kayser, Geschichte der deutschen Ballade, Berlin 1936, S. 199.

23 Leipzig o. J. (Max Brockhaus) 
konkrete autobiografische Situation, wohingegen op. 25, ähnlich wie die 1916 entstandene Vaterländische Ouvertüre Max Regers, als eine typische, durch den Ersten Weltkrieg motivierte nationalistische Erscheinung verstanden werden muss, als deren französisches Gegenstück etwa Henri Büssers Hymne à la France op. 57 auf einen Text Victor Hugos gelten kann. ${ }^{24}$ Pfitzner war der Überzeugung, „von eigentlicher Politik nicht das geringste“ zu verstehen und lediglich „die gefühlsmäßige nationale Einstellung “ 25 zu haben. Thomas Mann, der in Pfitzner „ein Bollwerk gegen die Zeitströmungen“ sah, „die ihn ästhetisch und politisch verunsicherten “, 26 scheint diese Einschätzung zu bestätigen, denn er kennzeichnete Pfitzner als „national, aber unpolitisch“. ${ }^{27}$ Der Krieg aber, so Mann, habe „die unausbleibliche Politisierung seines nationalen Empfindens " 28 bewirkt. Das Ergebnis, die, wie nicht zuletzt der Ad-libitum-Einsatz eines Männerchors zeigt, ganz auf Außenwirkung abzielenden Zwei deutsche[n] Gesänge, nimmt in Pfitzners Schaffen eine Sonderstellung ein, verkündet er, der im Palestrina Politik als Antagonistin der Kunst thematisierte, hier doch das erste und einzige $\mathrm{Mal},{ }^{29}$ wenn auch lyrisch verbrämt, eine politische Botschaft, wofür der große öffentliche Raum des Konzertsaals denkbar geeignet war. Die Lieder bedeuteten für Mann eine politische Stellungnahme eines „nationale[n] Künstler[s]“, der „sich zum antidemokratischen Nationalismus politisiert " ${ }^{30}$ hatte, womit Thomas Mann durchaus sympathisierte, denn mit Pfitzner verband ihn damals der Glaube, Deutschland kämpfe im Krieg um seine kulturelle Selbstverwirklichung, wobei die Musik - und hier sedimentiert sich einmal mehr die von Vaget betonte „Rolle der Musik in der Legitimationsstrategie des deutschen Imperialismus “31 - substantiell ist für die deutsche Identität: Pfitzner, so Mann „ersehnte den kriegerischen Triumph Deutschlands, widmete demonstrativ, als die Wogen des U-Boot-Streites am höchsten gingen, ein Kammermusikwerk [sic] dem Großadmiral von Tirpitz [...]“.32 Dass Thomas Mann von einem „Kammermusikwerk“ spricht, mag auf

24 Siehe Annegret Fauser, Der Orchestergesang in Frankreich zwischen 1870 und 192, Laaber 1994, S. 174.

25 Hans Pfitzner, Eindrücke und Bilder meines Lebens, in: Sämtliche Schriften, Bd. 4, hrsg. von Bernhard Adamy, Tutzing 1987, S. 594.

26 Hans Rudolf Vaget, Seelenzauber. Thomas Mann und die Musik, Frankfurt a. M. 2006, S. 208. In diesem Kontext ist auch an die Politisierung der Literatur z. B. durch Emile Zola und Heinrich Mann zu denken.

27 Thomas Mann, Betrachtungen eines Unpolitischen [1918], hrsg. und textkritisch durchgesehen von Hermann Kurzke (= Thomas Mann, Große kommentierte Frankfurter Ausgabe. Werke - Briefe - Tagebücher, hrsg. von Heinrich Detering u. a., Bd. 13.1), Frankfurt a. M. 2009, S. 462.

28 Ebd.

29 Trotz Pfitzners nationalistisch-antisemtischer Einstellung ist es m. E. eine erhebliche Fehleinschätzung, die Kantate Von deutscher Seele zu den „politisch intendierte[n] Werke[n]“ (Birgit Jürgens, „Deutsche Musik" - das Verhältnis von Ästhetik und Politik bei Hans Pfitzner [= Historische Texte und Studien 24], Hildesheim/Zürich/New York 2009, S. 137) zu zählen, verzichtet das Werk, romantische Topoi wie Einsamkeit und Vergänglichkeit thematisierend, doch auf jedes nationalistische Pathos. Der Titel, den Pfitzner möglicherweise der Volksliedsammlung Aus deutscher Seele des mit ihm befreundeten jüdischen Schriftstellers Ludwig Jacobowski entlehnte, rekurriert offenbar auf die Vorstellung, das deutsche Wesen sei romantisch (deshalb kann Rüdiger Safranski sein Buch zur Romantik „Eine deutsche Affäre“ nennen), was wiederum in der zeitgenössischen Überzeugung gründet, die Musik, von E. T. A. Hoffmann als „die romantischste aller Künste“ bezeichnet, sei von substanzieller Bedeutung für die deutsche Identität.

30 Mann, Betrachtungen eines Unpolitischen, S. 462.

31 Vaget, Seelenzauber, S. 14.

32 Mann, Betrachtungen eines Unpolitischen, S. 462. Alfred von Tirpitz war von 1897 bis 1916 Staatssekretär des Kaisers Wilhelm II. direkt unterstellten Reichsmarineamtes. Tirpitz, Mitbegründer des Deut- 
den ersten Blick verwundern, ist aber schnell und einfach damit zu erklären, dass er die für seine musikalische Sozialisation ganz wesentliche Gattung „Lied“ - und dies ist im Hinblick auf den oben erwähnten gattungsästhetischen Diskurs nochmals sehr erhellend - nur innerhalb des aus dem Elternhaus gewohnten Aufführungskontextes des Salons sehen konnte. ${ }^{33}$

Die beiden Gedichte lassen sich zum einen auf die historische Situation, zum anderen auf die Person von Tirpitz applizieren. Im Falle des Gedichts Der Trompeter von August Kopisch $^{34}$ (1799-1852) ist der Sachverhalt recht schlicht, kann die siebte Zeile der ersten Strophe des Gedichts („Das war ein Mann von Stahl, ein Mann von edler Art“) doch keinen Zweifel daran lassen, dass hier der Widmungsträger gemeint ist. Im Falle des 1815 erstmals publizierten Gedichts Klage aus der Feder Eichendorffs ist der Konnex nicht wesentlich komplexer. Das Gedicht steht in Verbindung mit der zeitgenössischen historischen Situation, was die von Eichendorff selbst im Untertitel angegebene Datierung „1809“35 verdeutlicht. Das „unrechte Regiment“, von dem im Gedicht die Rede ist, bezieht sich auf Napoleon, der nach der verlorenen Schlacht gegen Österreich bei Aspern im Mai 1809 drei Monate später bei Wagram nochmals siegte und erneut Wien besetzte. Pfitzner nutzt Eichendorffs Verse zum Verkünden seiner nunmehr politischen Überzeugung im Hinblick auf die innenpolitische Situation. ${ }^{36}$ Dass Birgit Jürgens hierin „einen Missbrauch des Dichters “ 37 sieht, ist angesichts der weit verbreiteten Instrumentalisierung Eichendorffs für deutschtümelnd-nationales Denken verständlich, aber im konkreten Zusammenhang doch eher ungerechtfertigt, zumal es sich um einen Text handelt, den auch Eichendorff in der Zeit der Befreiungskriege mit durchaus politischer Intention verfasst hat.

Während in Klage die Harmonik aufgrund ihrer nahezu ausnahmslosen Diatonik ebenso schlicht ist wie die Marschrhythmik, erschöpft sich in Der Trompeter die Musik nicht in reiner Äußerlichkeit, also im Geschmetter des Blechs und im Rasseln des Schlagwerks, sondern zeigt zumindest Ansätze von motivischer Kontingenz und aus dem Text resultierende motivische Bezugnahmen mit Verweischarakter, auf die an dieser Stelle allerdings nicht näher eingegangen werden soll.

schen Flottenvereins, in dem die „Pangermanisten“ ihren antisemitischen Ressentiments nachhingen, forcierte den Aufbau der deutschen Schlachtflotte. 1916 trat er aus Protest gegen eine Einschränkung des U-Boot-Krieges zurück.

33 Vaget, Seelenzauber, S. 48ff.

34 Der mit Eichendorff und Hoffmann von Fallersleben zum sog. „Breslauer Künstlerverein“ gehörende Kopisch studierte zunächst Malerei in Prag, Wien und Dresden, wandte sich dann aber zunehmend der Dichtung zu.

35 Gedichte von Joseph Freiherrn von Eichendorff, Berlin 1837, S. 145. Siehe hierzu: Joseph von Eichendorff, Werke in sechs Bänden, Bd. 1, hrsg. von Hartwig Schulz, Frankfurt a. M. 1987, S. 890f.

36 Robert Braunmüller, Deutsche Seelen. Pfitzners Kantate und die Eichendorff-Rezeption, in: Mitteilungen der Pfitzner-Gesellschaft 66 (2006), 14-29, hier: S. 23.

37 Jürgens, „Deutsche Musik“-das Verhältnis von Ästhetik und Politik bei Hans Pfitzner, S. 137. 
Lethe $^{38}$ ist eine Vertonung des gleichnamigen Gedichts von Conrad Ferdinand Meyer, dessen Texte bereits dem Liederzyklus op. 34 zugrunde lagen und, neben Dichtungen anderer Autoren, auch Eingang in die Chorphantasie Das dunkle Reich op. 38 (1929/30) gefunden haben. Die Dichtung wurde inspiriert durch das heute im Louvre befindliche allegorische Gemälde Le soir von Charles Gleyre, „das einen Dichter zeigt, dem die Leier entsinkt, während sich im Hintergrunde ein Boot mit elf Frauen und einem geflügelten Knaben vom Ufer entfernt, dem jener wehmütig nachschaut “. ${ }^{39}$ Die ganz auf Verinnerlichung angelegte Vertonung entstand als Reaktion auf den Tod der Ehefrau im Jahr 1926 im Kontext der o. g. Chorphantasie, der Oper Das Herz op. 39 und der neun Klavierlieder op. 40 und 41. „Ein treuer Hund legt sich aufs Grab und stirbt, ich komponierte das Lied ,Lethe'“, so kommentierte Pfitzner später die Entstehung.

Das schon aus Die Heinzelmännchen bekannte Prinzip, mittels deklamatorischer Auffälligkeiten semantische Details zu akzentuieren, begegnet in Lethe wieder, ist hier aber insofern noch wirkmächtiger, weil die nahezu durchgängige Achteldeklamation mit Hervorhebung akzent- und bedeutungstragender Silben durch punktierte Viertel Abweichungen umso mehr hervortreten lässt. Von besonderer Prägnanz ist die den dramatischen Höhepunkt der Dichtung darstellende 6. Strophe („Dir entriß in trotz'gem Liebesdrange / Ich die Schale, warf sie in die Flut, / Sie versank, und siehe, deine Wange / Färbte sich mit einem Schein von Blut“). Ein stürmischer Walzerrhythmus, der als Ausdruck des „trotz'gen Liebesdranges“ den ersten anderthalb Zeilen zugrunde liegt, mündet in einen mit einer Quartole vertonten Aufschrei („warf sie in die Flut“), dem, mit pausendurchsetzter Deklamation vorgetragen, der Kulminationspunkt der Traumvision („... und siehe, deine Wange / Färbte sich mit einem Schein von Blut") folgt, das Bild der wieder lebend geglaubten Geliebten.

Im Fokus der vierten Zeile der 2. Strophe („Eine Schale, draus ein jeder trank“) steht ganz das hier erstmals erwähnte Substantiv "Schale“, was durch die inmitten der Achteldeklamation umso auffälligere Dehnung der Akzentsilbe gleichermaßen wie durch den relativen Hochton mit nachfolgendem Oktavsprung unmittelbar evident wird. Dass Pfitzner an dieser Stelle gleichzeitig in die Versstruktur des Gedichts eingreift, das Ende der dritten Zeile („Kreisend durch die Reihe sah ich glänzen“), indem er vor „sah“ eine Pause einfügt, mit der vierten Zeile verbindet, so dass die letzten Worte der dritten Zeile („,sah ich glänzen“) ganz im Sog des folgenden Substantivs „Schale“ stehen, unterstreicht die große Bedeutung dieses Gefäßes, das den Trank des Vergessens enthält und einen nahezu sakralen Charakter zu besitzen scheint, erinnert es doch an den Erlösung verheißenden Kelch mit dem Blut Christi in der katholischen Liturgie. Diese Assoziation scheint die Vertonung der ersten Zeile der 5. Strophe „Die Reihe war an Dir zu trinken“ mit einer Folge, die Liszt als „tonisches Symbol des Kreuzes“ bezeichnet hat, zu bestätigen, wird doch auch hier, nun durch ein Fremdzitat und die quasi psalmodische Deklamation, der rituelle Charakter der Situation verdeutlicht, eine Überhöhung in das christliche Abendmahl vollzogen.

Innerhalb der ersten Zeile der 3. Strophe („Jetzt erscholl ein Lied voll süßer Wehmut“) springen die Zweierligaturen unmittelbar ins Auge. Im Zusammengehen mit der schlichten colla parte-Begleitung des Orchesters können sie als Ausdruck der um 1800 geltenden Bestimmungsmerkmale des Liedes, nämlich Einfachheit und Naivität, verstanden werden.

38 Berlin 1926 (Adolph Fürstner).

39 Dieter Borchmeyer, Letales Symposion: Conrad Ferdinand Meyers Lethe, in: Olaf Hildebrandt/Thomas Pitroff (Hrsg.), Auf klassischem Boden begeistert. Antike-Rezeption in der deutschen Literatur, Freiburg i. Br. 2004, S. [283]-288, hier S. 285. 
Solchermaßen eher lediglich Illustrierendes - als einziges weiteres Element sei die als Bild der durch die Reihe „kreisenden“ Schale verstehbare Pendelbewegung in den Fagotten ab dem 7. T. nach Buchstabe A genannt - sinkt in dieser Komposition aber ab zur Bedeutungslosigkeit. Vielmehr eignet der Musik ein symbolisches und verweisendes Meinen, das sich einerseits in der Harmonik, andererseits in Zitaten aus eigenen Werken und, wie eben schon gezeigt, Fremdzitaten manifestiert.

Der Anfang von Lethe offenbart einmal mehr ein Charakteristikum Pfitzner'scher Kompositionsweise, das ihn deutlich z. B. von Reger unterscheidet, nämlich die Trennung von komplexer Chromatik und schlichter Diatonik - letztere ist vornehmlich mit dem „Nachen ohne Ruder“ (1. Strophe, zweite Zeile) bzw. der „leise zieh'nden Barke“ (4. Strophe, dritte Zeile) verbunden - sowie ihr unvermitteltes Nebeneinanderstellen. Das Werk beginnt mit einem verminderten Septakkord, der bei enharmonischer Umdeutung des Tonmaterials als verkürzte Dominante mit None von E-Dur verstanden werden kann. Während des bereits erwähnten Höhepunkts der Traumvision innerhalb der 6. Strophe, als das lyrische Ich Leben in der vermeintlich Toten zu entdecken glaubt (, [...] deine Wange, Färbte sich mit einem Schein von Blut"), erklingt, vom nachfolgenden Verlauf durch eine Generalpause abgegrenzt und dadurch umso deutlicher akzentuiert, über vier Takte eben dieser E-DurAkkord, jene Tonart also, die offenbar, wie in Schuberts Der Lindenbaum oder in Verführung op. 33/1 von Richard Strauss, eine Traumatmosphäre, das Entrücktsein in eine glücklichere Vergangenheit symbolisiert. Die dominantische Korrelation vermag auf eine aus dem Spannungsverhältnis resultierende Sehnsucht nach der Vergangenheit, nach dem geliebten Wesen zu verweisen. Der Dominante ist nun eine motivische Wendung unterlegt, ein weiteres Fremdzitat, das unschwer als das nach einer fallenden Terz chromatisch weitergeführte "Schicksalsmotiv“ aus Wagners Ring zu identifizieren ist. Der es-Moll-Akkord, mit dem der Anfangston des Motivzitats harmonisiert ist, besitzt offenbar eine eigene semantische Dimension: Da er ebenso für das erste der Vier Lieder für eine Singstimme und Klavier op. 30/1 Pfitzners, einer Vertonung des mit der Anrufung „Lethe!“ beginnenden Gedichtes Sehnsucht nach Vergessen von Nikolaus Lenau, von zentraler Bedeutung ist, darf der Akkord als Chiffre der Sehnsucht nach Vergessen verstanden werden. Die Dechiffrierung dieser dem Orchester zugewiesenen und im Verlauf der Komposition leitmotivisch mehrfach wiederholten Konstellation von harmonischer Struktur und Zitat leistet ihr letztmaliges Erklingen nach der abschließenden Feststellung des lyrischen Ich's „Und ich wusst' es wieder, Du bist tot“, d. h.: Es gibt kein Vergessen. Der Tod des geliebten Menschen ist unumkehrbar. Dass hier das Adjektiv „tot“ nur von einem funeralen Kontexten vorbehaltenen Tamtam-Schlag im $p p p$ begleitet wird, unterstreicht die definitive Realität des Todes und zeigt damit einmal mehr den für die Gattung so typischen essentiellen Charakter der Instrumentation. Mit dem Ineinanderführen von Wagner-Zitat und fest umrissener akkordischer Struktur gelingt es Pfitzner, gleich zu Beginn in der Musik mehrere Ebenen zu verschränken, nämlich den im Gedicht formulierten Traum (Dominante von E-Dur), die Sehnsucht nach Vergessen (es-Moll-Akkord), gleichzeitig aber auch das Wissen des lyrischen Ichs um die Tatsache des nicht mehr ungeschehen zu machenden Todes des geliebten Menschen („Schicksalsmotiv“). Die Musik suggeriert also einen Bewusstseinszustand, den man im Kontext des Wagner'schen Diktums von „Ahnung und Erinnerung" sehen darf.

Bemerkenswert ist nun die Hinführung zum Höhepunkt der Traumvision, die Vertonung der dritten und vierten Zeile der sechsten Strophe. Der anfänglichen Walzerepisode - dass sie gleich zu Beginn von der das Werk einleitenden, semantisch hoch bedeutsamen Konstellation grundiert wird, verdeutlich umso mehr ihren Trugcharakter - schließt 
sich eine neuerliche, vom ursprünglichen harmonischen Kontext losgelöste Exposition des „Schicksalsmotivs“ in den tiefen Streichern an, der dreimal eine diastematische Variante des Motivs folgt. Die Vergrößerung aller Intervalle um eine kleine Sekunde (bei Buchstabe F und 4. T. nach Buchstabe F) bedeutet eine Transformation des Motivs, bewirkt sie doch seine Aufhellung und damit die Suspendierung seines Gehalts, die aber nurmehr transitorisch ist, ein Traum eben, wohingegen schon beim dritten Erklingen (7. T. nach Buchstabe F) das Orchester die Vision wieder der Realität zuführen zu wollen scheint, beschränkt sich die Augmentation der Intervallik hier doch nur auf das Anfangsintervall. Der neuen diastematischen Anlage korrespondiert eine gänzlich neue harmonische Situation. Ist die Ursprungsversion des "Schicksalsmotivs“ in einen komplexen harmonischen Kontext eingebunden, so ist seine Transformation Oberstimme von zwei pentatonisch zu verstehenden Akkordgebilden, also von zwei Akkorden, die in keinem funktionalen Verhältnis stehen, sondern einen reinen Farbwert haben und somit als Symbol des „färbte sich“, wie es in der Gedichtvorlage heißt, verstehbar sind.

Die von Pfitzner in einem Brief vom 24.10.1926 an Margitta Fischer selbst erwähnten Eigenzitate $^{40}$ sind für die Entschlüsselung des Gehaltes von Lethe von zentraler Relevanz. Das Erklingen des mit der Erscheinung Lukrezias, der verstorbenen Ehefrau Palestrinas, verbundenen Motivs aus dem 1. Akt ( $Z$ 167) des Palestrina nach der Textstelle „ich erkannte ... deine Stimme, die den Chor durchdrang " (ab Buchstabe C, Solo-Violine) ist ein Zitat von höchst prägnanter Referentialität: Indem Pfitzner auf die entsprechende Szenerie der Oper, auf die Vision Palestrinas rekurriert, dessen Schaffenskraft nach dem Tod seiner Gattin erlosch, gibt er sich selbst als lyrisches Ich zu erkennen. Während in Meyers Gedicht persönliche Erfahrungen allenfalls „symbolisch versetzt “ ${ }^{41}$ aufscheinen, ist die tote geliebte Frau in Pfitzners Orchesterlied eben nicht mehr nur bloße Fiktion, sondern, für Pfitzner gleichermaßen wie für seinen Opernhelden Palestrina, bedrohliche Realität. Ein weiteres Eigenzitat, den Worten „kühl sie war“ (4. Strophe, zweite Zeile) unterlegt, scheint dies zu bestätigen: Es handelt sich um die ersten vier, tristangesättigten Akkorde des Liedes Abbitte op. 29,1 auf einen Text von Friedrich Hölderlin. Ein Zweifaches ist hier von Bedeutung: Mit dem Zitat huldigt Pfitzner der geliebten Gattin, die im Hölderlinschen Text als „Heilig Wesen“ bezeichnet wird, und gleichzeitig ist es die Musik, die „weiß“, dass dem Tod, als dessen Chiffre die Kälte des Wassers fungiert, Realität eignet, denn das Gedicht Hölderlins ist keine Traumvision, sondern vom Wissen um ihren Tod geprägte Zwiesprache mit der Geliebten. Auf diese Weise wird in der Vertonung das Gedicht tatsächlich zu „Erlebnislyrik“ im Sinne Wilhelm Diltheys, der bekanntlich jedes Gedicht als sprachlichen Niederschlag persönlicher Erlebnisse des Verfassers verstand. Der Text liefert auf diese Weise die Grundlage für ein musikalisch gefasstes Stück Autobiographie. Das Lied ist also auch Medium der Selbstaussage, die, in der Gestalt eines Orchesterlieds präsentiert, eine öffentliche Bekundung bedeutet, die von existenzieller Betroffenheit zeugt und offenbar weniger gemein hat weder mit dem im Kontext der Zweiten Wiener Schule häufig zitierten Schlagwort der „öffentlichen „Einsamkeit “ 42 noch mit einer öffentlichkeitswirksamen Selbststilisierung.

40 Walter Abendroth, Hans Pfitzner, München 1935, S. 269.

41 Borchmeyer, Letales Symposion, S. 285.

42 Nikolaus Urbanek, „Öffentliche Einsamkeit“? Anmerkungen zum atonalen Liedschaffen Alban Bergs und Anton Weberns, in: Öffentliche Einsamkeit. Das deutschsprachige Lied und seine Komponisten im frühen 20. Jahrhundert, hrsg. von Michael Heinemann und Hans-Joachim Hinrichsen, Köln 2009, S. [95]121. 
Mit der in den Vordergrund gerückten Poetisierung des Begleitsatzes geht das Orchester in seiner Funktion als Träger des musikalischen Ausdrucks deutlich über die Klavierbegleitung in den Liedern Hugo Wolfs hinaus, so dass in Lethe das neudeutsche Prinzip der textexegetischen Vergegenwärtigung durch die Orchesterbegleitung zweifellos den gleichen Rang erreicht z. B. wie in Richard Strauss' Nächtlicher Gang. In den früheren Orchestergesängen Pfitzners liegt die Wahl des Orchesters offenbar nicht zuletzt in der Möglichkeit orchestraler Effekte begründet, wie sie Fauser schon für L'Egyptienne von Félicien David geltend gemacht hatte. ${ }^{43}$ D. h. die Musik ist eher „,tönende Illustration“ der Dichtung denn Sprache über der Wortsprache. ${ }^{44}$ Lethe dagegen lässt, da, um mit Schumanns Worten zu sprechen, die „Singstimme allein [...] nicht alles wirken, nicht alles wiedergeben “ ${ }^{45}$ kann, das „Gedicht mit seinen kleinsten Zügen im feineren musikalischen Stoffe “" 46 nachwirken. Mag in Die Heinzelmännchen z. B. das Orchester „mehr aus äußerem Schmuckbedürfnis“ erscheinen, so ergibt sich seine Wahl im Falle von Lethe aus der von Hausegger geforderten „inneren Notwendigkeit“. 47 Pfitzner erliegt hier nicht einmal ansatzweise der „Versuchung, auf Äußerlichkeiten, wie tonmalerische Illustration, bloßen Klangeffekt u. dergl. mehr Nachdruck zu legen“, 48 die Louis mit einer auf Verinnerlichung angelegten lyrischen Dichtung als nicht vereinbar ansieht. Mit Lethe, für die einmal mehr von Hauseggers These gilt, nur „ein ganz kultiviertes Publikum vermag die Musik in ihrem steten Zusammenhang mit der Dichtung verfolgen “, 49 hat Pfitzner einen Orchestergesang geschaffen, der nicht nur die ästhetische Höhe zeigt, die der Gattung zukommt, sondern zudem, ganz im Sinne Richard Wagners, ein „Wort-Ton-Kunstwerk“, in welchem in der Musik „der dichterische Gedanke zum unwillkürlich ergreifenden Gefühlsmomente " 50 wird und sich das „Sprachvermögen des Orchesters" 51 zum „Vermögen der Kundgebung des Unaussprechlichen" der „unsagbare“ Tod des geliebten Menschen - steigert.

Eine abschließende Bemerkung soll dem im Hinblick auf Pfitzners Denken über das Komponieren von Vokalmusik bedeutsamen produktionsästhetischen Aspekt von Lethe gelten. Unbestreitbar ist es hier der Text, der in Koinzidenz mit der konkreten Lebenssituation einen komplexen Schaffensprozess generieren konnte. Damit lieferte Pfitzner allerdings ein Beispiel für einen Typus von Liedkomposition, gegen den er selbst heftigst polemisiert hat. „Das Gros der Vokalmusik späterer und jetziger Zeit“, so Pfitzner, „füllt das zweite Gebiet. Da geht die Komposition vom Wort aus. In diesem Satz liegt das Gefährliche und Unmusikalische des Verfahrens. "53 Lethe führt diese These aber ad absurdum, denn mit Hilfe von Deklamation, Zitaten und harmonischer Konzeption fasst Pfitzner hier die Verknüpfung des Gehalts der Textvorlage und seiner autobiografischen Situation auf höchst artifizielle und ästhetisch überzeugende Weise in Musik, von der kaum vorstellbar ist, dass sie, wie man

43 Siehe Fauser, Der Orchestergesang in Frankreich, S. 29

44 Vgl. Jean Paul, Hesperus, in: Werke, hrsg. von Norbert Miller, Bd. 1, S. $776 f$.

45 Robert Schumann, in: NZfM 1843, Bd. 18, S. 120.

46 Ders., in: NZfM 1840, Bd. 13, S. 118.

47 Hausegger, Über den Orchestergesang, S. 211.

48 Louis, Die deutsche Musik der Gegenwart, S. 237.

49 Hausegger, Über den Orchestergesang, S. 210.

50 Richard Wagner, Oper und Drama; ders., Dichtungen und Schriften, hrsg. von Dieter Borchmeyer, Bd. 7), Frankfurt a. M. 1983, S. 293.

51 Ebd., 321.

52 Ebd., 329.

53 Hans Pfitzner, Die neue Ästhetik der musikalischen Impotenz, in: ders., Gesammelte Schriften, 2, Augsburg 1926, S. 211. 
es z. B. von Reger kennt, schon vor der Dichtung existierte. Insofern ist der Begriff „,absolute" Musik den Rudolf Stephan für manche der Lieder Pfitzners wohl zu Recht in Anspruch nahm, ${ }^{54}$ auf Lethe keinesfalls applizierbar.

Die Musikgeschichtsschreibung hat sich von manchen ideologischen Verengungen zu befreien begonnen. Qualifizierende Kategorienpaare wie Fortschritt und Restauration sind als Hilfsmittel der Geschichtskonstruktion allmählich in den Hintergrund getreten, um den Blick auf die Vielfalt der Konzepte und Stile freizugeben, was nicht zuletzt auch einen unbefangeneren Umgang mit dem Konservativen Hans Pfitzner ermöglicht, natürlich die Akzeptanz der These vorausgesetzt, dass die Person nicht das Werk kompromittiert, seine geistige Kumpanei mit dem Nationalsozialismus also, sein kruder Antisemitismus und das bestenfalls mit Naivität und Altersstarrsinn erklärbare Festhalten an alten Feindbildern und Ideologien auch noch nach Kriegsende außen vor bleiben. Es eröffnet sich dann die Möglichkeit, auch in seinem Werk zumindest punktuell und annäherungsweise der Zeitgenossenschaft zwar nicht mit der neuen Musik - wann die zu beginnen habe, ist nach wie vor nicht geklärt ${ }^{55}$-, zumindest aber mit der modernen Musik gewahr zu werden. Denn trotz des streitbaren Wagnerianers Pfitzner bekanntermaßen ausdrücklicher Ablehnung der Moderne, die sich in den Invektiven gegen Ferrucio Busoni und Paul Bekker niedergeschlagen hatte, finden sich in Lethe Sedimente des Bewusstseins für den historischen Standort. Unübersehbar ein Wort-Ton-Kunstwerk im Sinne Richard Wagners, realisiert dieser Orchestergesang gleichzeitig, wenn auch als verspäteter Gattungsbeitrag, in paradigmatischer Weise ein „dialektisch verschränktes Streben nach äußerer Monumentalität und innerer Differenzierung “56, das Hermann Danuser als ein Charakteristikum der Ästhetik der Moderne verstand.

54 Rudolph Stephan, Sind Hans Pfitzners Lieder „Absolute“ Musik?, in: Hans Pfitzner und die musikalische Lyrik seiner Zeit. Bericht über das Symposion Hamburg 1989, hrsg. von Wolfgang Osthoff, Tutzing 1994, S. 221-236.

55 Siehe hierzu Siegfried Mauser, Musikalische Moderne und Neue Musik als kompositionsgeschichtliche Paradigmen, in: Siegfried Mauser/Matthias Schmidt (Hrsg.), Geschichte der Musik im 20. Jahrhundert (= Handbuch der Musik im 20. Jahrhundert, Bd. 1), Laaber 2005, S. 31-39.

56 Hermann Danuser, Die Musik des 20. Jahrhunderts (= NHbMw 7), Laaber 1984, S. 13. 